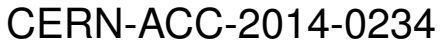 \\ EuCARD-2
}

Enhanced European Coordination for Accelerator Research \& Development

\section{Scientific Report}

\section{Performance tests of prototype high field HTS coils in Grenoble}

\author{
Miyoshi, Y (NIMS-ICYS) et al
}

19 September 2014

\section{EUCARD ${ }^{2}$}

The EuCARD-2 Enhanced European Coordination for Accelerator Research \& Development project is co-funded by the partners and the European Commission under Capacities 7th Framework Programme, Grant Agreement 312453.

This work is part of EuCARD-2 Work Package 10: Future Magnets (MAG).

The electronic version of this EuCARD-2 Publication is available via the EuCARD-2 web site $<$ http://eucard2 . web.cern.ch/> or on the CERN Document Server at the following URL:

$\langle$ http://cds.cern.ch/search?p=CERN-ACC-2014-0234 > 


\title{
Performance tests of prototype high field HTS coils in Grenoble
}

\author{
Yasuyuki Miyoshi, Arnaud Badel, Xavier Chaud, Tara Benkel, Benjamin Vincent, Pascal Tixador, Julien Marpaud, \\ François Debray, Thibault Lécrevisse, Mélanie Devaux, Philippe Fazilleau, and Jean-Michel Rey
}

\begin{abstract}
Longitudinal and transverse quench propagation tests were performed at LNCMI high field test facility on instrumented double pancake coils fabricated by CEA-Saclay using co-wound HTS tapes. Energy deposited on an embedded heater initiated a quench and its subsequent propagations. Following the resulting thermo-mechanical analysis, a conductor design with stacked HTS tapes co-wound with stabilizers was conceived. A 10-turn demonstrator racetrack coil has been fabricated from the stacked HTS conductor with an aim to investigate the operation margin of an HTS coil in a background magnetic field misaligned from the coil axis as expected for the operating condition of an accelerator magnet insert.

A test set up with a high current capacity up to $3 \mathrm{kA}$ and angular variability that utilizes a room temperature $\varnothing 376 \mathrm{~mm}$ bore $10 \mathrm{~T}$ resistive magnet at LNCMI in Grenoble has been built. The performances and operation margin of the racetrack coil were investigated.
\end{abstract}

Index Terms-High-field magnet, High-temperature superconductors (HTS), HTS coils, HTS quench propagation,

\section{INTRODUCTION}

$\mathrm{T}$ HE generation of high magnetic field by HTS, beyond the conventional low temperature superconductors (LTS) effectiveness, is a technological challenge that is attracting accelerated efforts today. The industrial scale improvement of coated conductors in both transport and mechanical properties is especially promising, and has already encouraged large scale applications to invest in the realization of the HTS

Automatically generated dates of receipt and acceptance will be placed here; authors do not produce these dates. This work was supported in part by the French Agence Nationale pour la Recherche under the SUPERSMES project, the European Commission under the FP7 Research Infrastructures projects EuCARD and EMFL (European Magnetic Field Laboratory). This work is part of EuCARD Work Package 7: Super-conducting High Field Magnets for higher luminosities and energies. (Corresponding author: Arnaud Badel.)

Y. Miyoshi was, as an EMFL invited researcher, with the University Grenoble Alpes / CNRS / INSA / UPS, LNCMI, 38042 Grenoble, France. He is now at NIMS-ICYS, 1-2-1 Sengen, Tsukuba, Ibaraki 305-0047 Japan, email: MIYOSHI.Yasuyuki@nims.go.jp.

A. Badel, T. Benkel and P. Tixador are with the University Grenoble Alpes / CNRS, G2Elab / Institut Néel, 38042 Grenoble, France, e-mail: arnaud.badel@grenoble.cnrs.fr, pascal.tixador@grenoble.cnrs.fr.

B. Vincent, J. Marpaud, F. Debray and X. Chaud are with the University Grenoble Alpes / CNRS / INSA / UPS, LNCMI, 38042 Grenoble, France, email: benjamin.vincent@lncmi.cnrs.fr, $\quad$ francois.debray@lncmi.cnrs.fr, xavier.chaud@lncmi.cnrs.fr.

T. Lécrevisse, M. Devaux, P. Fazilleau and J.-M. Rey are with the CEADSM-IRFU-SACM, 91191 Gif sur Yvette cedx, France, e-mail: philippe.fazilleau@cea.fr, j-m.rey@cea.fr. magnet technology [1]-[6]. Within the EuCARD European Project, there was activities aimed to investigate the feasibility of a $6 \mathrm{~T}$ HTS insert in a $13 \mathrm{~T} \mathrm{Nb3Sn}$ dipole [7] that now continues with the project EuCARD2 [8]. As participating members of the consortium, we have carried out HTS insert design studies that involve electromagnetic load and quench protection model analysis [9]-[11] as well as experimental investigation of quench events in small prototype HTS coils to validate the thermal model [12].

There are engineering activities on the HTS SMES [13][15] in Grenoble as a pulsed current source for railgun applications. With high strength HTS coated conductors the mechanical strength margin is no longer the first limiting factor in SMES design [15]. The major concern now is the protection that is governed by the time taken to reach the maximum allowed temperature. For this reason, we are investigating the quench dynamics and co-wound shunt for current sharing by testing on small prototype pancake coils, which will also be critical for HTS insert project at LNCMI.

Based on the same methodological approach, we will describe two separate experiments on small HTS prototype coils carried out using the LNCMI facilities [16]-[18] by the three collaborating parties CEA-Saclay, G2Elab/Institut Néel, and LNCMI. We report firstly the test carried out at $\varnothing 170 \mathrm{~mm}$ bore $19 \mathrm{~T}$ site on a heavily instrumented double pancake coil to measure the quench propagation at various operating conditions, in the interest of establishing a coil winding and instrumentation method and investigating quench dynamics. Secondly, we report the first test carried out on a small scale racetrack coil to investigate the coil performance margin as a function of coil orientation with respect to the external field using a new $3 \mathrm{kA}$ probe in a $\varnothing 376 \mathrm{~mm} 10 \mathrm{~T}$ bore.

\section{QUENCH PROPAGATION IN HTS PANCAKE COIL UNDER MAGNETIC FIELD}

The recently developed concept of SMES based on YBCO coated conductors [14], [15] targets a specific energy density of $20 \mathrm{~kJ} / \mathrm{kg}$ to improve the current record on the existing SMES design. The high current density and the low quench propagation velocity lead to concerns about protecting the magnet. For this purpose, we have wound prototype HTS coils with quench propagation measurement instrumentations, i.e. a quench heater embedded in the coil winding and an array of voltage taps in its vicinity. The high current test probe, the high speed data acquisition techniques, the construction of a prototype coil ("HTS2") and the measurement results from 
another coil have been reported earlier [12], [17]. We report here the outcome of the recent quench propagation tests on HTS2 performed in Grenoble.

\section{A. Quench propagation experiment: sample preparation}

An assembly of one double pancake coil sandwiched between two single pancake coils were fabricated by CEASaclay using SCS4050-AP tapes from SuperPower Inc. with 2 $\times 20 \mu \mathrm{m}$ of copper coated shunt, and a half of the central double pancake coil was heavily instrumented with an embedded quench heater and voltage taps in the winding (Fig. 1). The single pancakes consist of two conductors cowound with superconducting side facing each other, in order to increase the current carrying capacity to account for the less favorable magnetic field orientation at the ends of the coil assembly. For all pancakes, the conductors were co-wound with a $150 \mu \mathrm{m}$ extra copper stabilizer insulated on the outer face by a $30 \mu \mathrm{m}$ polyester tape. The coil dimension and winding turns are shown in table I.

A set of voltage taps has been implemented within the instrumented pancake; this set allows to measure the velocity of the resistive zone, longitudinally and transversally. The entire coil assembly is mounted on a large heat exchanger which temperature is regulated by heating elements and a calibrated Cernox ${ }^{\mathrm{TM}}$ cryogenic temperature sensor. There are copper thermalizing layers inserted in between the pancakes to ensure temperature uniformity. Another calibrated Cernox ${ }^{\mathrm{TM}}$ sensor is mounted on the coil assembly itself to monitor the sample temperature.

\section{B. Quench propagation experiment: experimental method}

The coil assembly mounted on a measurement probe is inserted into a variable temperature cryostat that fits into the $\varnothing$ $170 \mathrm{~mm}$ bore $19 \mathrm{~T}$ site at LNCMI. Current is ramped in the coil and set at the desired value for typically several seconds. The temperature is monitored using cernox ${ }^{\circledR}$ sensors to ensure that it remains stable. While on this plateau the embedded

TABLE I

HTS2 COIL PARAMETERS

\begin{tabular}{ll}
\hline \hline Diameters & ID: $86 \mathrm{~mm}$ OD: $110 \mathrm{~mm}$ \\
Single pancake turns & 33 \\
Double pancake (instrumented half) & 41.5 \\
Double pancake (non-instrumented half) & 43.5 \\
Total conductor length & $67 \mathrm{~m}$ \\
\hline \hline
\end{tabular}

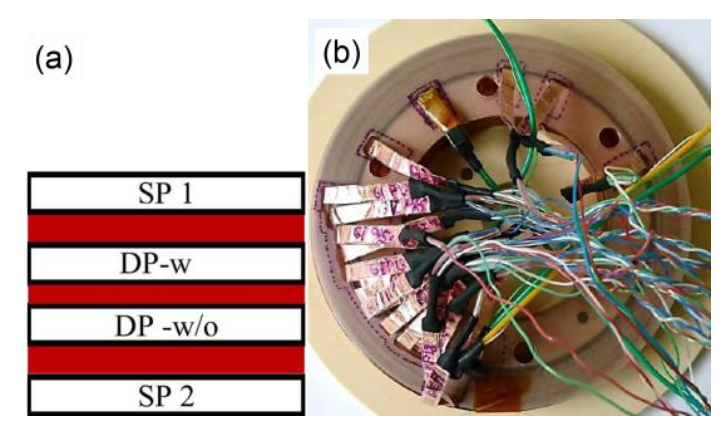

Fig. 1. (a) A schematic of the HTS2 construction, i.e. double pancake (DP with/without instrumentations) sandwiched by two single pancake (SP) coils, with thermalizing copper in-between, and (b) the top view of heavily instrumented pancake coil (DP-w) for quench propagation test. heater is powered, the deposited energy is evaluated from the current pulse and the voltage drop across the heater. Note that we discuss hereafter the minimum quench energy (MQE) in terms of this heater energy, but naturally it includes dissipations to all bodies in thermal contact with the heater. The instrumented coil voltages are monitored in real-time, and the internal processor of our data acquisition system (DAQ) monitors the coil voltages differentially (e.g. comparing the overall voltage of the instrumented half and non-instrumented half of the double pancake) in order not to overstep a certain voltage criterion defined by the normal state coil resistivity. When this safety condition is met, the DAQ sends a signal to the current source for an emergency shutdown. In this way, the coil current is under control when enough energy has been deposited for a quench. The safety condition is software controlled by the threshold voltage and the temporal window allowed to overstep this threshold.

\section{Quench propagation experiment: experimental results}

The coil assembly passed successfully $120 \mathrm{~A}$ at $4.2 \mathrm{~K}$ and $14 \mathrm{~T}$ [12]. Initially, attempts were made to propagate the quench in liquid helium bath at $4.2 \mathrm{~K}$ in $7 \mathrm{~T}$ background field. As it became apparent that quench attempts in liquid helium resulted in hot spot and no propagation, we decided to continue the experiment in gas helium.

In gas helium at $10 \mathrm{~K}$, in background field $8 \mathrm{~T}$ and at coil current $280 \mathrm{~A}$, we measured the MQE to be $6.8 \mathrm{~J}$. As we show in Fig. 2, clearly at $6.8 \mathrm{~J}$ the coil recovers and at $6.97 \mathrm{~J}$ the coil quenches. As discussed above, this energy includes dissipations to environment. At this MQE, we observed both longitudinal and transverse quench propagations.

For the longitudinal propagation, at the conductor in direct contact to the heated element, one sees the heater pulse initiates a resistive transition which is self-sustaining that eventually reaches a quench. The neighboring voltage taps also register resistive voltages before the safety measure shuts down the coil current. The longitudinal propagation velocity of $45 \mathrm{~mm} / \mathrm{s}$ is calculated from the time quench takes to reach the two neighboring sections. The slow propagation at 10 20 times reduced velocity compared to LTS is a well-known characteristic of HTS and it poses a serious issue of quench

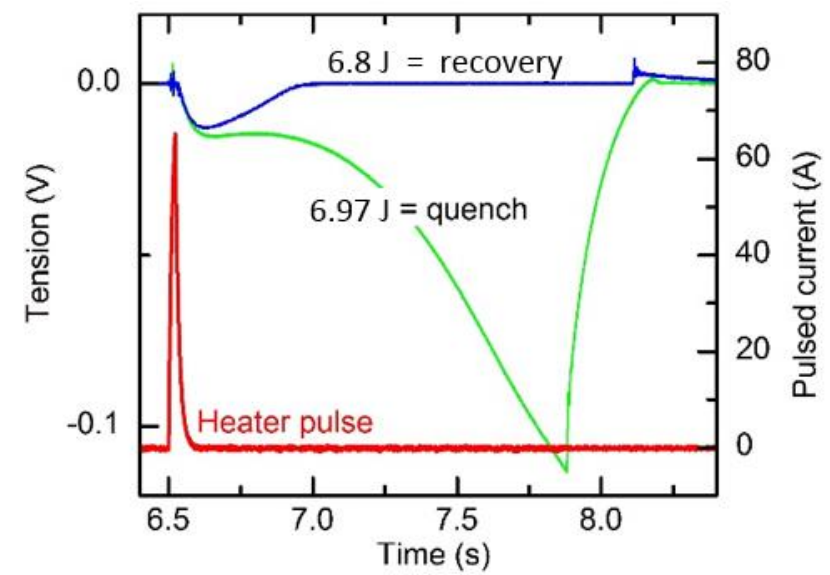

Fig. 2. Illustration of $\mathrm{MQE}$ at $\mathrm{T}=10 \mathrm{~K}, \mathrm{~B}=8 \mathrm{~T}$, Icoil $=280 \mathrm{~A}$. The heater pulse (red) initiates a disturbance which recovers (blue) or grows to a quench (red). 
detection difficulty. The transverse propagation velocity evaluated here is $126 \mathrm{~ms} / \mathrm{turn}$, which is a time taken to propagate to the neighboring turn.

\section{HIGH CURRENT HTS RACETRACK COIL TEST}

The feasibility of HTS dipole insert magnet is being investigated to reach $20 \mathrm{~T}$ within $13 \mathrm{~T} \mathrm{Nb}_{3} \mathrm{Sn}$ dipoles in view of the foreseen LHC upgrading program High Energy LHC (HE-LHC) [8]. We describe test runs on a prototype racetrack coil, wound from a high current conductor consisting of stabilizer co-wound coated conductors, in a newly constructed $3 \mathrm{kA}$ class high current probe as a function of sample orientation with respect to the external field. The primary goal here is to check the nominal operation of the coil with external magnetic field oriented at 10 degrees to the conductor surface.

\section{A. High current HTS racetrack coil test: sample preparation}

The conductor design chosen for this test (Fig. 3) consists of two $12 \mathrm{~mm}$ wide coated conductors with the superconducting layer sides co-wound with a $70 \mu \mathrm{m}$ copper stabilizer sandwiched and soldered in between, and with a 100 $\mu \mathrm{m} \mathrm{CuBe} e_{2}$ ribbon with a $30 \mu \mathrm{m}$ polyester insulation layer on the exterior side [11]. The copper stabilizer is for thermal stability and $\mathrm{CuBe}_{2}$ is to withstand the high Lorentz load expected in the operating condition of an insert magnet to a high field dipole at LHC, i.e. about $600 \mathrm{MPa}$. For this first test, a small racetrack coil of 10 turns was fabricated by CEASaclay. The objective of this test coil is to experimentally validate the electrical and mechanical HTS insert coil design, obtain data that is necessary for the modelling feedback, and to develop the necessary winding technique. The coil winding is equipped with a heater, inserted between the $2 \mathrm{nd}$ and $3 \mathrm{rd}$ winding, matching the one side length of the racetrack, with ability to choose the length of the heated element. There are six voltage taps placed on the conductors: at the beginning of the turn; at the 3rd winding at the beginning of the quench heater; at the 3rd winding after the quench heater; and at each subsequent turns. The quench heater and voltage taps are not for measuring the quench propagation velocity, but to detect any appearance of resistive voltage, which may or may not lead to a quench, owing to the input heater energy. This input energy then is considered as a minimum operating margin.

(a)

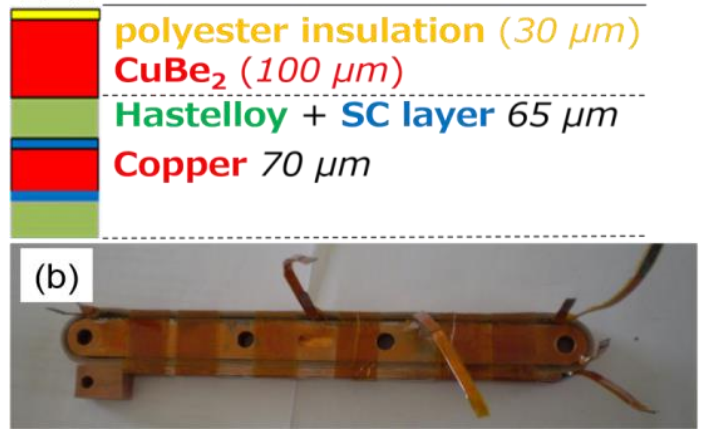

Fig. 3. (a) Schematic of the conductor construction and photographs of the (b) wound racetrack coil.

\section{B. High current HTS racetrack coil test: test probe}

The nominal operating current of the HTS dipole insert is $2800 \mathrm{~A}$ in $13 \mathrm{~T}$ background field [11], and so a test environment is required that can supply current up to $3 \mathrm{kA}$ at $4.2 \mathrm{~K}$ in liquid helium bath, with external magnetic field in a large enough bore to contain the coil mounted on a test probe. The test environment chosen was a $376 \mathrm{~mm}$ bore $10 \mathrm{~T}$ Bitter magnet available at LNCMI. The probe design (Fig. 4a) was constrained by the geometry of this magnet and the existing cryostat with inner diameter of $298 \mathrm{~mm}$ that fits into it. The upper part of the current leads is constructed out of two concentric copper tubes. Below the level of liquid helium the current leads are continued by custom-made high current HTS elements to reduce the boil off due to heat leak and Joule heating. The current leads at the sample end are in circular clamp form (or "copper shells") with slots (Fig. 4b) at every 1 degree up to 10 degrees angle and another at 20 degrees that enable to rotate and fix the sample angular orientation against the background field while at room temperature. The sample experiences a large Lorentz load from carrying a high current in high field, and the resultant torque is supported by the stainless steel flange which is mechanically fixed to a thick G10 tube (not shown in the Fig. for clarity) that continue up to the probe top flange. The probe is equipped with temperature sensors as well as voltage taps along the current leads and near the sample to monitor the operation of the current leads.

The experimental procedure is similar to the test described in section II, with current ramping of the coil followed by plateau several seconds long, during which the heater is energized to bring energy to the winding and initiate the quench. The deposited energy is calculated from the heater voltage and current. The coil safety is observed in real time as in the double pancake experiment, but since no symmetry exists in this racetrack coil, the difference between two different winding turns is monitored with a numerical factor to balance the inductive signals. When the differential coil
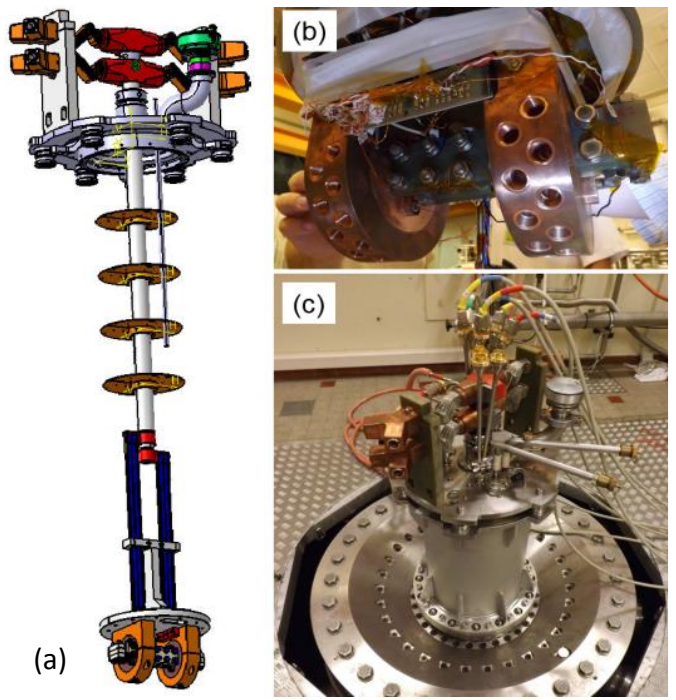

Fig. 4. (a) The newly constructed high current variable angle probe schematic, (b) a view of mounted coil, and (c) the probe ready for test inserted into the large bore magnet. 
voltage oversteps a threshold voltage over a temporal window, a signal is sent to the current source for an emergency shutdown. Due to the high operating current and the low ramp rate for coil safety, the measurement duration is long and some duplicate coil signals are observed in real time by multichannel differential oscilloscope.

\section{High current HTS racetrack coil test: experimental results}

At the beginning of the measurement campaign, it became apparent that the coil had been damaged by the winding process. At a close inspection, it was revealed that at one corner of a winding turn, one tape of the stacked conductor had been damaged which may have reduced the current transport capacity of the coil. For this reason, the coil performance was checked step by step, starting from lower current of $800,1000,1200$, and finally $1400 \mathrm{~A}$ with $0^{\circ}$ orientation of coil axis with respect to the background field 9.5 T. The coil successfully carried these currents, despite the presence of some ohmic voltage, without any sign of a quench. The experiment was reproduced successfully at $9.5 \mathrm{~T}$ $10^{\circ}$ orientation. We investigated the operating margin of this coil by energizing the heater element. An example of this measurement is illustrated by Fig. 5. The coil current was ramped up to $1.2 \mathrm{kA}$ while the coil voltages were monitored differentially in real-time by the DAQ in case of abnormal coil voltage appearance. As the coil current is held constant, there was a background resistive voltage of $1 \mathrm{mV}$ possibly due to the dissipation at the damaged part of the coil but without any sign of heating further degrading the coil performance within the timeframe of the experiment. Then the heater was energized in this case at the instance of $16.6 \mathrm{~s}$ measurement time. The heater energy was varied by changing the input current amplitude or the duration of heater pulse. In the Fig. 5, the heater pulse is indicated by the hatched regions which resulted in the deposition of $6.8 \mathrm{~J}$ and $8.3 \mathrm{~J}$ in the two cases.

In case (a), there was some resistive voltage but the coil recovered as the pulse ended, while case (b) is when the rapid growth in resistive voltage lead to the coil current shutdown for safety. After the shutdown, the coil performance was

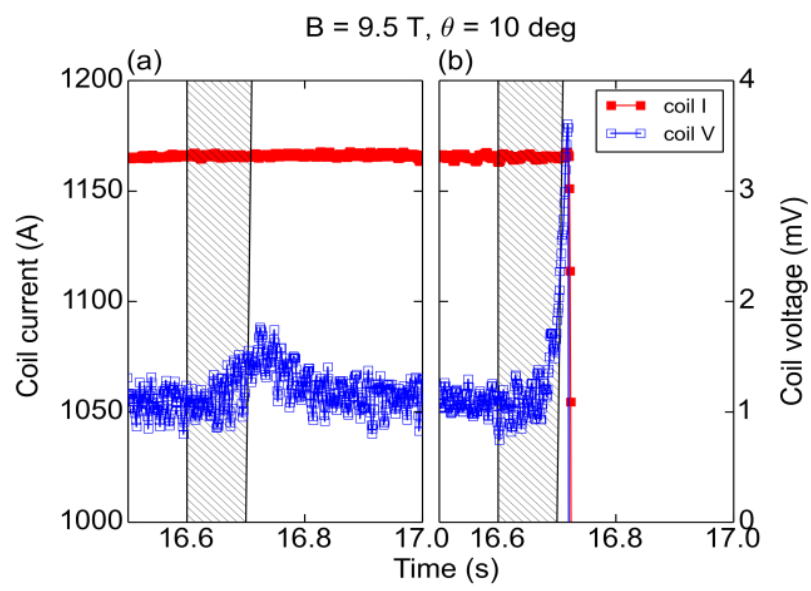

Fig. 5. At background field $9.5 \mathrm{~T}$ and coil orientation 10 degrees, measured coil voltage at the instance of heater pulse, indicated by the hatched region, for the cases with deposited heater energy of (a) $6.8 \mathrm{~J}$ that recovered and (b) $8.3 \mathrm{~J}$ that triggered the coil current shutdown are shown. checked again with the lower heater energy to confirm the integrity of the coil and the operational margin in terms of input heater energy. The same measurement procedure was repeated at different coil currents and at different orientations.

\section{SUMMARY}

We have successfully wound double pancake coils and performed quench propagations under high magnetic field. A controlled quench method with fast data acquisition has been developed and quench propagations both along and across the winding layers have been measured. Although not presented in this paper, a number of duplicate experiments have been performed with modified measurement configurations for the detailed inspection of various experimental aspects. The most recent challenge of winding a high current HTS racetrack coil in view of expected upgrade of LHC with HTS inserts has been carried out, despite some damage present in the coil. The racetrack coil has been tested under high field with orientation as expected in the insert dipole magnet. The tests will resume with a new racetrack coil with a full nominal current to complete the experimental effort. From these preliminary results, it can be concluded that both solenoid and racetrack HTS coils show very large stability margins so that quenching is unlikely under normal operation current. The detection and protection against quench events may therefore not be a major issue for HTS coils. Protecting the coils is however critical in over-current condition, when the current reaches a value higher than the locally-defined critical current. This case will be studied in future works, it can happen especially in case of induced current due to the quench of an LTS outsert coil. In such case, the detection of the over-current condition should be easy from the monitoring of the outsert coil so that effective protection of HTS insert is still feasible.

\section{ACKNOWLEDGMENT}

The authors would like to thank CEA technical support team for coils parts manufacturing. They also thank technical support from the Institut Néel and LNCMI for the fabrication of sample holder and cryostat, particularly $\mathrm{C}$. Bruyère, $\mathrm{O}$. Tissot and J.-M. Tudela. They are grateful to J. Aubril and E. Yildiz for their help during the coil tests at LNCMI.

\section{REFERENCES}

[1] H. Song, P. Brownsey, Y. Zhang, J. Waterman, T. Fukushima and D. Hazelton, "2G HTS Coil Technology Development at SuperPower", IEEE Trans. Appl. Supercond. vol. 23, no. 3, Jun. 2013, Art. No. 4600806.

[2] U. Floegel-Delor, T. Riedel, D. Wippich, B. Goebel, R. Rothfeld, P. Schirrmeister, F. N. Werfel, A. Usoskin and A. Rutt, "Long-Length Coated Conductor Copper Plating Fabrication", IEEE Trans. Appl. Supercond. vol. 23, no. 3, Jun. 2013, Art. No. 6602204.

[3] N. Zangenberg, G. Nielsen, N. Hauge, B. R. Nielsen, A. Baurichter, C. G. Pedersen, L. Bräuner, B. Ulsøe and S. P. Møller, "Conduction Cooled High Temperature Superconducting Dipole Magnet for Accelerator Applications", IEEE Trans. Appl. Supercond. vol. 22, no. 3, Jun. 2012, Art. No. 4004004

[4] N. Amemiya, K. Goda, H. Otake, T. Nakamura, T. Ogitsu, K. Koyanagi, T. Kurusu, Y. Mori, Y. Iwata, K. Noda and M. Yoshimoto, "Progress of Research and Development of Fundamental Technologies for 
Accelerator Magnets Using Coated Conductors", IEEE Trans. Appl. Supercond. vol. 23, no. 3, Jun. 2013, Art. No. 4601905.

[5] R. Gupta, M. Anerella, A. Ghosh, P. Joshi, H. Kirk, S. L. Lalitha, R. Palmer, W. Sampson, P. Wanderer, H. Witte, Y. Shiroyanagi, D. Cline, A. Garren, J. Kolonko, R. Scanlan and R. Weggel, "High Field HTS Solenoid for a Muon Collider-Demonstrations, Challenges, and Strategies", IEEE Trans. Appl. Supercond. vol. 24, no. 3, Jun. 2014, Art. No. 4301705 .

[6] H. W. Weijers, W. D. Markiewicz, A. J. Voran, S. R. Gundlach, W. R. Sheppard, B. Jarvis, Z. L. Johnson, P. D. Noyes, J. Lu, H. Kandel, H. Bai, A. V. Gavrilin, Y. L. Viouchkov, D. C. Larbalestier and D. V. Abraimov, "Progress in the Development of a Superconducting $32 \mathrm{~T}$ Magnet With REBCO High Field Coils", IEEE Trans. Appl. Supercond. vol. 24, no. 3, Jun. 2014, Art. No. 4301805.

[7] G. de Rijk, "The EuCARD high field magnet project", IEEE Trans. Appl. Supercond. vol. 22, no. 3, Jun. 2012, Art. No. 4301204.

[8] http://eucard2.web.cern.ch/activities/wp10-future-magnets-mag

[9] M. Devaux, F. Debray, J. Fleiter, P. Fazilleau, T. Lécrevisse, C. Pes, J. M. Rey, J. M. Rifflet, M. Sorbi, A. Stenvall, P. Tixador and G. Volpini, "HTS Insert Magnet Design Study", IEEE Trans. Appl. Supercond. vol. 22, no. 3, Jun. 2012, Art. No. 4203605.

[10] E. Härö, A. Stenvall, T. Lécrevisse, J. Fleiter, J. M. Rey, M. Sorbi, M. Devaux, C. Trophime, P. Fazilleau, G. Volpini, P. Tixador, F. Hornung and C. Pes, "Quench Considerations and Protection Scheme of a High Field HTS Dipole Insert Coil", IEEE Trans. Appl. Supercond. vol. 23, no. 3, Jun. 2013, Art. No. 4600104.

[11] J. M. Rey, M. Devaux, F. Bertinelli, X. Chaud, F. Debray, M. Durante, G. Favre, P. Fazilleau, T. Lécrevisse, C. Mayri, C. Pes, F. Pottier, M. Sorbi, A. Stenvall, P. Tixador, J. M. Tudela, T. Tardy and G. Volpini, "HTS Dipole Insert Developments", IEEE Trans. Appl. Supercond. vol. 23, no. 3, Jun. 2013, Art. No. 4601004.

[12] T. Lécrevisse, X. Chaud, F. Debray, M. Devaux, P. Fazilleau, F. P. Juster, Y. Miyoshi, J. M. Rey, P. Tixador and B. Vincent, "Quench Propagation in YBCO Pancake: Experimental and Computational Results", IEEE Trans. Appl. Supercond. vol. 23, no. 3, Jun. 2013, Art. No. 4601805.

[13] A. Badel, P. Tixador, P. Dedié, "Test of a twin coil HTS SMES for high power pulse operation". IEEE Trans on Appl. Supercond., vol. 21, pp. 1375-1378, June 2011.

[14] P. Tixador, N. T. Nguyen, J. M. Rey, T. Lecrevisse, V. Reinbold, C. Trophime, X. Chaud, F. Debray, S. Semperger, M. Devaux and C. Pes, "SMES Optimization for High Energy Densities", IEEE Trans. Appl. Supercond. vol. 22, no. 3, Jun. 2012, Art. No. 5700704.

[15] B. Vincent, P. Tixador, T. Lecrevisse, J. M. Rey, X. Chaud and Y. Miyoshi, "HTS Magnets: Opportunities and Issues for SMES", IEEE Trans. Appl. Supercond. vol. 23, no. 3, Jun. 2013, Art. No. 5700805.

[16] X. Chaud, F. Debray, L. Ronayette, E. Mossang, P. Brosse-Maron, A.-J. Vialle, P. Tixador, J.-M. Rey, T. Lecrevisse and C.-E. Bruzek, "Characterization of YBCO Coated Conductors Under High Magnetic Field at LNCMI", IEEE Trans. Appl. Supercond. vol. 22, no. 3, Jun. 2012, Art. No. 6600704.

[17] Y. Miyoshi, X. Chaud, F. Debray, B. Vincent, P. Tixador, T. Lecrevisse, J. M. Rey, H. Oguro, S. Awaji, K. Watanabe, G. Nishijima and H. Kitaguchi, "HTS Coil Test Facility in a large Bore 20 T Resistive Magnet at LNCMI", IEEE Trans. Appl. Supercond. vol. 23, no. 3, Jun. 2013, Art. No. 9500204.

[18] F. Debray, J. Dumas, C. Trophime, and N. Vidal, "DC High Field Magnets at the LNCMI", IEEE Trans. Appl. Supercond. vol. 22, no. 3, Jun. 2012, Art. No. 4301804. 